\title{
30 Jahre Psychoanalytisches Seminar Zürich: Institutionalisierung/Des-Institutionalisierung?
}

\author{
Interview mit Sylvia von Arx (SvA), Olaf Knellessen (OK), \\ Monika Leuzinger (ML), Peter Passett (PP); Fragen: Emilio Modena
}

Journal: Ich möchte mit einer Frage an Peter Passett beginnen. Da wir ein Jubiläumsjahr haben 2007, 30 Jahre PSZ, und da du von Anfang an dabei warst, damals noch an der Tellstrasse, wollte ich dich fragen, ob du das Seminar noch wiedererkennst? Was hat sich deiner Meinung nach verändert?

PP: Ja natürlich erkennt man etwas wieder, wenn man ständig dabei ist. Aber wenn ich einen 30-jährigen Winterschlaf gemacht hätte und wieder aufgewacht wäre, würde ich es wahrscheinlich nicht mehr erkennen, weil sich doch sehr vieles geändert hat. Natürlich nicht die Psychoanalyse. Die Psychoanalyse ist dieselbe, aber die Psychoanalyse ist etwas, was interpretationsbedürftig ist. Heute sind die Akzente dieser Interpretation am Psychoanalytischen Seminar sehr anders als vor 30 Jahren. Damals hat man ganz stark die Idee der Dissidenz in den Vordergrund gestellt, nicht so zu sein, wie alles, was es damals schon gab. Insbesondere natürlich nicht wie das alte Seminar der schweizerischen Gesellschaft, aber auch in Bezug auf die herrschenden Verhältnisse, auf die Gesellschaft rundherum wollte man etwas Anderes, etwas Neues verwirklichen und gleichzeitig auch etwas Subversives, etwas Revolutionäres, etwas vielleicht Dekonstruktives. Heute geht es vor allem darum, dabei zu sein, mitzumachen, so zu sein, wie die anderen sind, wie die anderen therapeutischen Ausbildungen, so wie das Gesundheitswesen eine solche Institution haben will. Es geht darum, sich zu legitimieren, zu zeigen, dass man auch so ist, wie man sein sollte. Das ist ein völlig neuer, oder im Vergleich zum Alten völlig anderer Charakter.

Journal: Darfich geradezudir überleiten, Monika, weildu in der Seminarleitung bist und all diese institutionellen Veränderungen begleitest. Was ist heute notwendig, damit dem PSZ die staatliche Legitimation erhalten bleibt?

ML: Ich bin mir nicht sicher, ob das so stimmt, dass sich das ganze Seminar verändern und sich neu einrichten muss, weil von aussen etwas Neues kommt, nämlich diese 
Psychotherapie-Weiterbildung. Ich plädiere nach wie vor dafür, die zwei Gebiete zu trennen. Man kann das alte Seminar und die alten Ideen beibehalten und für das Neue, das geschaffen werden muss, eine neue juristische Form finden.

\section{Journal: Das ist deine persönliche Position.Aber wie steht es mit der Mehrheit im PSZ, welche Schritte stehen an, um die staatliche Anerkennung zu bekommen?}

ML: Wir vermischen ständig die Ausbildung und das Betreiben der Psychoanalyse einerseits und die Weiterbildung in Psychotherapie anderseits. Das sind für mich zwei Sachen, die sehr unterschiedlich sind und nicht zusammengehören. Und wenn du jetzt fragst, was notwendig ist: Natürlich wird für die Weiterbildung in Psychotherapie von aussen, vom Kanton, resp. der Charta, eine gewisse Regulierung verlangt. Da müssen Verträge geschaffen werden usw. Das muss, das müssen wir machen. Ein Regelwerk sozusagen.

Journal: Sylvia, in Zusammenhang mit diesen Institutionalisierungsschritten, die ja absehbar waren, hast du die Initiative ergriffen, eine Institutionsanalyse am PSZ durchzuführen. Es haben schon mehrere Sitzungen einer Grossgruppe stattgefunden, an der sich ungefähr 50 TeilnehmerInnen beteiligen. Kannst du mir erklären, was deine damit verbundene Absicht oder deine Hoffnung war? Was zeichnet sich in dieser nun vorankommenden Institutionsanalyse $a b$ ?

SvA: Ich habe diese Idee nach einem dieser häufigen Abende an der TV gehabt, als ich wieder einmal den Eindruck gewonnen hatte, dass es stagniert. Ich hatte einfach genug von dem ewig Gleichen. Ich fürchtete, dass es sich nicht mehr bewegen lässt ... Beim Überlegen, was da überhaupt abläuft, bin ich als Analytikerin darauf gekommen, dass man vielleicht auch hier, in der Institution, mit unseren Mitteln schauen könnte, wie es sich mit den Identifizierungen und Vorstellungen verhält, die wir mit dem PSZ verbinden. Ich finde es wichtig, wenn man versucht, über die Phantasien auch die unbewussten Prozesse zu erfassen, die in dieser Institution ablaufen. Ich selber habe mit Grossgruppen keine Erfahrung, so hat es mich auch ganz persönlich interessiert, wie man das macht, wenn man die eigene Theorie und Technik auf die Gruppe anwendet.

\section{Journal: Hast du einen ersten Eindruck?}


SvA: Meine Hoffnung, dass sich etwas weiter bewegt, hat sich schon ein Stück weit erfüllt. Aber es harzt damit, wirklich auf eine Ebene zu kommen, wo über Identifikationen gesprochen werden kann. Es geht sehr oft um Sachzwänge, was vielleicht auch am Zeitgeist liegt; trotzdem finde ich, es hat sich etwas bewegt. Ich habe die Empfindung, dass man im Verlauf dieser drei Sitzungen besser über Inhaltliches reden konnte, losgelöst von den festgefahrenen Positionen, die sonst immer wieder von einzelnen Leuten verkörpert werden. Auch wenn man mit dem Prozess nicht einverstanden war, blieb man doch mehr bei den Inhalten. Das zeichnet sich ab. Das würde eigentlich meine Hoffnung erfüllen, aber es ist harzig.

Journal: Spiegelt sich in dieser Grossgruppe etwas vom Vereinsleben oder vom Gruppenleben des Seminars?

SvA: Ich glaube schon - es ist eine lebendige Gruppe! Trotzdem ist sie schwierig. Ich glaube auch nicht, dass sich mögliche Veränderungen bereits schon in der Gruppe zeigen werden. Wahrscheinlich wird sich das erst im Nachhinein zeigen, in dem, was weiter mit dem PSZ passiert.

Journal: Eine Frageandich, Olaf:Du bisteinerseits in derTeilnehmerversammlung einer der Kritiker dieser neuen Regelung für die analytische Psychotherapie gewesen, die ja verschiedene Institutionalisierungsschritte mit sich bringt, wie Qualifikationsgespräche, wie auch die Notwendigkeit einer Vereinsgründung etc. Anderseits bist du in der Ressortgruppe Öffentlichkeitsarbeit, welche die grossartige Idee lanciert hat, zum 30-jährigen Jubiläum des PSZ einen wissenschaftlichen Preis zu stiften. Wie erlebst du dich in dieser doch irgendwie ambivalenten Position? Auf der einen Seite die Fundamentalkritik, auf der anderen das aufbauende Weiterwirken?

OK: Ich erlebe das nicht als unvereinbaren Widerspruch. Für mich ist das PSZ von allem Anfang an mit einer Diskussionskultur verbunden, die sich ständig um die Widersprüche und Gegensätze bemüht, die es innerhalb der Psychoanalyse, die es aber auch innerhalb des Seminars gibt (das ist ja auch von Parin schon in der Absichtserklärung so formuliert worden). Das gehört zum Kern dieser Organisation, auch der organisatorischen Struktur des Seminars. Meiner Meinung nach hat sich das bislang bewährt. Das sieht man auch daran, dass bei uns eine Vitalität herrscht, eine Kreativität und Produktivität, um die man uns rundum beneidet. Wir waren neulich in Deutschland, und es war erschütternd zu sehen, dass dort so 
etwas überhaupt nicht vorkommt. Das ist das, wofür ich mich engagiere. Das ist mit ein Grund, weshalb ich verschiedene Punkte an dem Vorschlag für eine neue Weiterbildung kritisiert habe. Ich hab versucht, diesen Vorschlag an dem Anspruch zu messen, den Geist und die Tradition des Psychoanalytischen Seminars auch in dieserWeiterbildung aufrecht zu erhalten. Ich bin damit kaum durchgedrungen. Es wurde sogar darüber abgestimmt, ob ich nun drei oder vier Anträge stellen darf, das ist ein Novum, das fand ich gar nicht «lustig» ... Aber auch das Kritisieren solcher Fragen ist Teil meines Engagements, und ich fühle mich mit dem PSZ verbunden. Ich habe den Eindruck, da eine gute Ausbildung in Psychoanalyse gemacht zu haben. Ich fühle mich wohl mit dem PSZ und bin deshalb vor drei/vier Jahren, als es darum ging, sich um Öffentlichkeitsarbeit zu bemühen, in diese Ressortgruppe eingetreten. In der Ressortgruppe Öffentlichkeitsarbeit ist schon seit längerem die Idee entstanden, diesen Preis zu stiften. Das 30-jährige Jubiläum des Bestehens des PSZ schien uns wirklich ein guter Anlass zu sein, diesen Preis jetzt auch zu lancieren. Das haben wir mit Unterstützung des PSZ realisieren können, und so, wie's aussieht, wird dieser Preis auch weiterhin institutionalisiert.

Was mir bei diesem Preis weiterhin von Bedeutung war, ist, dass er auf eine interdisziplinäre Zusammenarbeit der Psychoanalyse mit anderen Disziplinen ausgerichtet ist, jeweils einer anderen Disziplin. Und das scheint mir ein wichtiger Punkt, um die Psychoanalyse aus der Sackgasse herauszuführen, in der sie auch bei uns am Seminar manchmal gerät, wenn sie sich allzu ausschliesslich auf die Klinik und auf die Psychotherapie ausrichtet. Der Bezug auf die Gesellschaftstheorie, die Kulturtheorie, war immer ein ganz wichtiger Teil unseres Selbstverständnisses und ist es immer noch. Das ist in dieser interdisziplinären Zusammenarbeit, auf die der Preis ausgerichtet ist, mit enthalten. Insofern ist diese Positionierung des Preises für mich eine Dokumentation oder ein Ausdruck dessen, was den Geist des PSZ in diesen 30 Jahren ausgemacht hat.

Journal: Nun hataber Petergesagt, dass sich dieser Geist wesentlich veränderthabe. Kann dieser kritische Geist weiter bestehen, wenn die Institutionalisierung derart fortschreitet, dass zum Beispiel eine einfache Gesellschaft nicht mehr tragbar ist und eineVereinsstrukturnotwendigwird.Dass dem Staatzu liebe Qualifikationsgespräche durchgeführtwerden müssen, Verträge mitWeiterbildungsexperten abgeschlossen werden und so weiter?

PP: Monika hat die Trennung von Ausbildung zur Psychoanalyse einerseits, die zum alten PSZ gehört, und Psychotherapie-Weiterbildung anderseits sehr betont. 
Das ist ein Versuch, der mir an sich sehr sympathisch ist. Aber das PSZ umschliesst nach wie vor beides. Es ist nicht getrennt. Und du, Sylvia, sprichst von Identität und von Institution, eine Institution sollte offenbar auch eine Identität haben, und die Leute in der Institution sollten eine Identität haben und in dieser Identität muss offenbar beides drin stecken. Du, Olaf, hast vor allem etwas angesprochen, was für das alte Seminar sehr charakteristisch war, ein Ort des Konfliktes zu sein. Also ein Ort, wo viele Konflikte Platz hatten. Wir hatten ja ursprünglich solche Metaphern, die sicher alle irgendwie zu kurz greifen, ein «freier Haufen», eine «Brüder- oder Schwesternhorde», ein «wilder Haufen» und all das. Aber das sind keine Institutionen! Solche Haufen haben auch keine Identität, darin gibt es Menschen, die ihre eigene Identität haben, die ein Verhältnis zu diesem Haufen haben. Und das ist nun eben die Veränderung, dass wir jetzt offenbar ungefragter Weise eine Institution geworden sind, eine Identität haben müssen, aber wiederum in dieser Institution Dinge vertreten, die offensichtlich nicht so gut miteinander unter einen Hut zu bringen sind.

ML: Diese Frage ist für mich noch wirklich offen, ob wir eine solche Institution werden sollen und werden müssen. Ich begebe mich jetzt ganz auf die pragmatische Ebene und nehme Olaf als gutes Beispiel für Öffentlichkeitsarbeit. Da gab es ein Interesse, diesen Preis zu kreieren und zu stiften und was machen sie? Sie gründen einen Verein. Aus diesem Haufen heraus hat sich eine Gruppe gefunden, die hat ein gemeinsames Interesse, sie sind durchaus verbunden mit dem PSZ, und machen etwas Eigenes. Weshalb kann man mit dieser Weiterbildung dieses Eigene auch nicht so gestalten, einen eigenen Verein für diejenigen, die die PsychotherapieWeiterbildung machen wollen und das ganze PSZ bleibt der wilde, unkontrollierte Haufen, wie du ihn vorhin genannt hast?

PP: Theoretisch kann man das. Und ich würde mich auch sehr freuen, wenn es gelingen würde. Aber die Frage ist, wie die Schwerpunkte liegen werden; man versucht, etwas mehr oder weniger Unmögliches doch noch möglich zu machen. Es ist schon heute so, wenn jemand von aussen über das PSZ redet, dann redet man im Grunde genommen von dieserWeiterbildung. Man redet von diesem Curriculum und darüber, ob wir anders als die Uni oder irgendwelche andere Weiterbildungsmöglichkeiten strukturiert sind. Ob es da Vor- und Nachteile gibt, und dieses Ursprüngliche, die Psychoanalyse-Ausbildung, wird immer mehr zum Anhängsel und meiner Ansicht nach schlussendlich zum Feigenblatt. Das finde ich peinlich. Ich möchte nicht, dass die Psychoanalyse das Feigenblatt wäre, sondern das Hauptanliegen! Dann 
könnte man nebenbei meinetwegen auch Psychotherapie-Ausbildung betreiben oder irgendetwas, aber ich glaube nicht, dass die Verhältnisse so sind, sie haben sich umgedreht.

\section{Journal: Sylvia, siehst du das auch so, dass die Psychoanalyse verschwindet?}

SvA: Ich muss mich dagegen verwahren, zur Feigenblatt-Vertreterin gemacht zu werden wegen meiner Haltung zur Institution, was ich vorhin gesagt habe. Was mir ein Anliegen ist, ist genau diese Möglichkeit, dass Verschiedenes möglich ist. Es ist ein Versuch, was jetzt gemacht wird, wobei von aussen viel Druck gekommen ist. Es ist ein Versuch, beides unter dem Dach des Seminars zu vereinigen. (Ich habe übrigens nachgesehen, wie sich die Begriffe «Seminar» und «Institut» unterscheiden: ein Seminar ist nämlich eine vorbereitende Bildungsanstalt, das wird heute im schulischen oder akademischen Bereich verwendet, kommt aber von einer Baumschule her. Beim Institut, heisst es einfach Forschungs- und Bildungsanstalt. Ich finde, das sagt schon einiges ...) (lacht) Mein Anliegen wäre, dass das Seminar nicht in diesen Querelen stagniert, sondern einen Versuch wagt. Mit dem, was wir abgestimmt haben, ist es ist jetzt angelegt, aber das kann jederzeit rückgängig gemacht werden. Dass deswegen die Psychoanalyse abhanden kommen könnte, befürchte ich auch, das befürchten wir alle. Aber wir müssen uns einsetzen, dass das nicht geschieht! Der Versuch ist noch nicht gemacht, dass wir die Psychoanalyse, so wie wir sie verstehen, an unserem Seminar beibehalten und weiterverfolgen neben so einer Enklave.

PP: «Wir können es wieder rückgängig machen!», dieses Argument höre ich jetzt seit 16 Jahren.

SvA: Vielleicht nicht rückgängig machen, aber wir können weitergehen in diesem Prozess.

PP: Man hat gesagt: «Wir gehen einmal in die Charta, wir können ja dann wieder austreten.

ML: Könnte man. Ja.

PP: Nein, wir könnten nicht mehr! 
ML: Doch. Wir könnten ein Ausstiegsszenario schaffen, wenn wir das wollten.

PP: Ja, rein theoretisch, aber es war doch etwas ganz anderes, als man nicht drin war. Da hätte man draussen bleiben können, wofür ich und viele votiert haben, eine eigenständige Haltung bewahren. Man hat gesagt: «Nein, wir gehen einmal rein, wir schauen.» Jetzt sind wir drin. Wenn wir jetzt austreten würden, hätte das eine ganz andere Bedeutung. Dann wären wir die Abtrünnigen, diejenigen, die nicht mehr wollen, die sich nicht der Vernunft beugen. Wir wären dann die Scharlatane, die in die Ecke Getriebenen. Mir wäre es immer noch recht, aber du weisst genauso gut wie ich, dass wir für einen solchen Austritt niemals mehr eine Mehrheit an diesem Seminar bekommen.

\section{SvA: Das ist richtig!}

PP: Rein theoretisch kann man immer. Aber praktisch ist dieser Ausstieg nicht möglich. Dasselbe gilt für die Psychotherapie-Ausbildung: wenn wir das einmal so gekoppelt haben, dann bleibt es eben so.

OK: So stimmt das nicht! Man hat lediglich über diese Weiterbildungsordnung abgestimmt, über den Vorschlag von Monika noch nicht. Ich halte ihn auch nicht für sehr glücklich. Ich bin nicht dafür und bin auch nicht der Meinung, dass es eine Parallele gibt zur Situation von der Ressortgruppe und dem Preis, weil wir einen Verein gegründet haben. Wir haben den Verein gegründet aus rein technischen Gründen, nicht etwa deswegen, weil wir irgend etwas ausserhalb des PSZ damit machen wollten. Wenn man hingegen einen Verein gründen würde, der zuständig wäre für dieWeiterbildungsordnung und für die Organisation dieserWeiterbildung, dann wäre das etwas ganz grundlegend Anderes. Man versucht nämlich einen Widerspruch und auch eine Differenz zu konstruieren zwischen der Psychoanalyse und der Psychotherapie, die es zwar gibt, es sind aber auch nicht einfach nur zwei grundsätzlich verschiedene Sachen. Das muss man schlicht und einfach auch mal sagen (Zustimmung aller). Das gilt auch für die Identität. Ich glaube, wir müssen aufpassen, es gibt immer die Gefahr zu polarisieren, Dichotomien herzustellen zwischen Identität und Differenz. Zum Beispiel bin ich durchaus mit dem Seminar identifiziert, aber gleichzeitig gibt es ganz viele Punkte, wo ich auch in deutlicher Differenz zu der mehrheitlichen Meinung des Seminars stehe. Das ist, was ich meine: Das gehört zum Leben und zu der Art und Weise, wie das Seminar bisher existiert hat und, wie ich auch glaube, weiterexistieren sollte. Was auch seine Lebendigkeit 
ausmacht, dass genau diese Fragen eben nicht auseinander dividiert werden. Und deswegen bin ich auch nicht dafür, einen Verein zu gründen, der dann sozusagen für die Psychotherapie zuständig wäre, und der Rest ist dann die Psychoanalyse mit der Illusion, würde ich sagen, dass man sich dann endlich mal mit der Psychoanalyse beschäftigen kann, weil man das Andere irgendwie los geworden ist. Das kann auf beide Seiten hin nicht funktionieren.

ML: Aber das ist doch eigentlich dasselbe. Die Weiterbildung wurde im Seminar beschlossen, wie der Preis auch, und ihr habt eine Struktur gebraucht, aus finanziellen, aus technischen Gründen, und ebenso braucht diese Weiterbildung aus technischen Gründen eine Form, oder?

OK: Nein, das ist überhaupt nicht dasselbe.

ML: Ich finde es einfach nicht notwendig, die Form, die dieseWeiterbildung braucht, über das ganze Seminar zu stülpen. Wir haben ja schon Erfahrung mitWeiterbildung. Zum Beispiel machen die Ärzte seit Jahr und Tag ihre Psychotherapie-Weiterbildung bei uns am PSZ. Sie haben ihre eigene Institution, die wird von der FMH getragen. Bei den Psychologen ist es anders, dieWeiterbildung wird bei ihnen nicht von einem Verein ausserhalb übernommen, sondern die wird uns übertragen. Aber das heisst doch nicht, dass wir das ganze Seminar danach richten müssten.

SvA: Peter und Olaf, ihr beide habt an einer Sitzung der Institutionsanalyse gesagt, dass ihr euch bewusst seid, oder dass ihr auch seht, dass eine Weiterbildung in irgendeiner Form in Angriff genommen werden muss. Oder institutionalisiert (habt ihr sicher nicht gesagt!), aber dass es ... oder habe ich mich da verhört?

PP: Also manchmal bin ich nicht mehr meiner Meinung, aber das würde mich jetzt sehr überraschen, wenn ich das gesagt hätte.

OK: Ich habe es gesagt, obwohl man mir immer wieder vorgeworfen hat, ich wolle das Ganze nicht, was schlicht und einfach nicht stimmt. Es gab eineVernehmlassung. In derVernehmlassung habe ich ein Papier geschrieben zu dieser Frage. Am Schluss des Papiers steht explizit, schwarz auf weiss, dass ich nicht dagegen bin, sondern dass ich finde, dass man das macht, ABER, dass man in Bezug auf die Ansprüche von aussen nur das erfüllt, was wirklich gefordert wird! Und nicht immer schon drüber hinaus geht! Und diese Forderungen auch auf eine Art und Weise zu erfüllen 
versucht, dass das, worum es uns bislang am PSZ gegangen ist, möglichst weitgehend erhalten bleibt. Das ist das, was ich geschrieben habe. Und das ist auch der Grund, weshalb meine Anträge bei dieser letzten TV gekommen sind, weil ich der Meinung bin, dass in diesem Vorschlag sehr viele Punkte enthalten sind, die weit über das hinaus gehen, was gesetzlich im Moment absehbar oder bereits gefordert ist! Es ist zum Beispiel gesetzlich nirgends erforderlich, dass man eine Unterscheidung macht zwischen - wie es noch im ersten Entwurf hiess - Vorklinik und Klinik. Später hat man das in «Basis und Aufbau» umgewandelt, was inhaltlich genau das Gleiche ist. Es ist ganz offensichtlich, dass sich dieses Modell an das Studium der Medizin anlehnt, mit dem wir von der Psychoanalyse her nichts zu tun haben. Ausserdem wurde gesagt, das werde auch bei anderen Instituten so gemacht. Es ist nur die Frage, was bringt es uns, wenn wir uns zum Beispiel am Studium der Medizin orientieren. Was bringt es uns, wenn wir uns am Freud-Institut orientieren. Ich bin der Meinung, es bringt uns überhaupt nichts! Und es steht im Widerspruch zu dem Anspruch, der formuliert ist: «Wir wollen eine solche Weiterbildung im Sinne und in der Tradition des PSZ machen.» Ich meine, wir sind in einer Situation, in der man sich halt auch an die gesellschaftlichen oder gesetzlichen Vorschriften orientieren muss, aber man muss versuchen, so weit wie möglich unsere Position zu wahren. Wir sind nach wie vor das grösste Weiterbildungsinstitut in der Schweiz. Und auch die Psychoanalyse hat nach wie vor eine Bedeutung, auch wenn diese während der letzten dreissig Jahre geschmälert worden ist. Ich meine, wir sollten unser Gewicht in die Waagschale werfen.

SvA: Ich bin auch der Meinung, dass man sehr aufpassen muss, aber vorhin ging es doch darum, ob man in der heutigen Situation überhaupt etwas machen muss! Peter sagt, man könne diesen Ballast nicht mehr abwerfen - aber was wäre die Alternative? Die Alternative wäre doch, dieses immer wieder heraufbeschworene Wort von der Trennung, oder nicht? Du sagst, man kann das nicht rückgängig machen: Wenn man es nicht mehr möchte, gäbe es eine Spaltung, so interpretiere ich, was du gesagt hast. Oder ist es nicht vielmehr so, dass man sagen muss, wir haben im Kern dieses Weiterbildungskonzept angenommen, und es kann wieder geändert werden. Zum Beispiel solche Aufteilungen, wie Basis und Aufbau, das kann bestimmt wieder geändert werden. Ich finde es nicht illusorisch, dass man so was ändert, wenn man neben der Psychotherapie-Weiterbildung die Psychoanalyse weiter pflegt und ihr auch wieder mehr Platz einräumt. 
PP: Ich habe vorhin schon gesagt, theoretisch ist das alles möglich. Ich halte es aber nicht für wahrscheinlich, und ich denke vielleicht etwas anders: Ich sehe, dass es grosse Entwicklungen gibt, die nicht nur mit unserem Seminar zu tun haben, die mit der Welt zu tun haben. Da gibt es grosse Ströme gegen die man sich nur sehr bedingt stellen kann. Nun glaube ich, dass im Moment der Strom ganz in Richtung Psychotherapie geht, und dass die Psychoanalyse in gewissem Sinn marginal wird. Ich glaube nicht, dass deswegen die Psychoanalyse untergeht. Mein persönliches Szenario ist so, dass ich denke, in etwa 10, 15, wahrscheinlich eher 20, 30 Jahren wird dieser ganze Schwindel auffliegen. Man wird merken, dass das Illusionen waren, was man unter Effizienz und all diesen Schlagwörtern gemeint hat, und dann wird sich wieder ein Bedürfnis nach dem, was Psychoanalyse einmal war, einstellen. Es ist allerdings eine grosse Gefahr, dass dann ganz andere Inhalte, zum Beispiel Esoterik, diesen Platz besetzen. Aber es wird sicher wieder eine grössere Aktualität für die Psychoanalyse geben. Meine Tendenz wäre gewesen, dass man in Kauf nimmt, dass man kleiner wird, dass man marginaler wird. Was die Frage der Psychotherapie betrifft, da bin ich auch der Meinung, dass man das nicht einfach abspalten kann. Analyse war immer auch Psychotherapie. Aber sie hat eine ganz spezielle psychotherapeutischeVorstellung. Freud, ein Aufklärer, hatte den Gedanken, dass, wenn man mehr wisse, das immer schon besser sei, dass das Therapie an sich ist. Wenn man mehr weiss, ist das schon im gewissen Sinne eine Heilung. Das ist meiner Ansicht nach der spezifisch therapeutische Ansatz der Psychoanalyse. Die moderne Psychotherapie und Psychotherapie-Forschung hat einen ganz anderen Ansatz. Die will objektivierteWirkungen sehen und die misst sie, dabei lügt sie sich ständig in die Tasche, aber sie misst die. Das hat mit diesem Wissen gar nichts zu tun, denn das wissen wir ja alle, Psychoanalysen verlaufen nicht gradlinig. Das Wissen eines Menschen über sich selbst und über seine unbewussten Motive kann sich vertiefen, aber er kann dabei immer depressiver werden. Er kann dabei sozial immer mehr von der Rolle fallen, und dann kann man sich fragen: «War das jetzt eine gute Therapie oder war es eine schlechte?» Aber psychoanalytisch könnte man, auch wenn diese messbaren Indizes ganz negativ verlaufen, immer noch von einem positiven Prozess sprechen. Und ich wäre immer dafür gewesen, dass man darauf bestanden hätte und gesagt hätte: «Wir biedern uns nicht mit diesen Therapievorstellungen an, wie man objektive Verbesserungen erzielt» (die man meiner Meinung nach gar nicht gezielt erreichen kann, die ergeben sich aus ganz anderen Gründen). Was ich beklage, ist derVerlust dieses Kernes. Ich bin trotzdem immer noch mit diesem Seminar identifiziert, aber dann geht es auch wieder um Differenz. Ich bin wahrscheinlich mehr mit dem identifiziert, was ich mir immer 
noch vom Seminar wünschte und nicht so sehr mit dem, was es ist. Das ist auch eine Identifikation.

SvA: Ich bin in ganz Vielem deiner Meinung. Man muss sich auch fragen, wie es mit den verschiedenen Generationen am Seminar steht. Glaubst du, dass das, was du jetzt über die Psychoanalyse gesagt hast, deshalb aus dem Unterricht oder aus den Seminarien verschwindet, weil diese Regelungen getroffen worden sind? Das tönt so, wie wenn plötzlich, wie durch ein Vakuum, alles unserWissen und Wollen, unsere Art die Psychoanalyse zu vertreten, heraus gesogen würde. Können wir das nicht trotz diesen Regelungen, die von aussen kommen, aufrecht erhalten? Ich glaube, man kann Psychoanalyse und Psychotherapie nur formal trennen, aber inhaltlich - das wollen wir ja nicht inhaltlich trennen! Ich glaube, das wollte auch niemand von der Akkreditierungsgruppe. Glaubst du nicht, dass es möglich ist, diese Inhalte der Psychoanalyse, die wir vertreten, weiterhin zu pflegen und zu vermitteln? Darüber müssen wir diskutieren.

Journal: IchseheeineSchwierigkeit,dieüberunsereeigenenInstitutionalisierungsschritte hinausgeht und die mit der ganzen gesellschaftlichen Entwicklung verbunden ist, dass nämlich überall immer weniger hochfrequente Psychoanalysen gemacht werden. Insbesondere für die jüngeren KollegInnen wird es immer schwieriger, Analysen (im Sinne von hochfrequenten, Freud'schen Analysen) durchzuführen. Und da frage ich mich, ob wir durch die Übernahme einer Weiterbildung in psychoanalytisch orientierter Psychotherapie gewissermassen nicht diese schlechte Realität zementieren, so dass es in 10 Jahren vielleicht gar keine hochfrequente Psychoanalysen mehr gibt?

OK: Ich glaube nicht, dass man unsere Position von Psychoanalyse nicht mehr vertreten könnte. Dazu haben wir eine zu lange Tradition, auch wenn jetzt solche Bestrebungen ganz offensichtlich da sind. Aber die gab's immer schon, das muss man auch sagen, z. B. den Status von Dozenten oder von Lehranalytikern einzuführen, das ist nichts Neues. Darüber haben wir auch schon früher abgestimmt. Früher liefen die Abstimmungen allerdings mehr in die andere Richtung ... Und wenn sich die Weiterbildung z. B. auch daran orientiert, wie die anderen Institute solche Ordnungen machen, dann heisst das deswegen noch nicht, dass wir jetzt genauso werden, wie diese Institutionen, wie diese anderen Institute. Dazu sind wir immerhin schon 30 Jahre zusammen, das lässt sich nicht einfach über den Haufen werfen. Was mich mehr irritiert, ist, weshalb man das überhaupt anstrebt, unsere 
Weiterbildung so zu gestalten, wie die anderen. Wieso sollen wir unsere Eigenständigkeit aufgeben und damit auch die Attraktivität, die nach wie vor damit verbunden ist? Das bringt, wenn man das jetzt rein markttechnisch anschaut, überhaupt keinen Vorteil. Mal ganz abgesehen davon, dass auch Psychotherapie-Weiterbildung für uns immer mit einem bestimmten Verständnis von Psychoanalyse verbunden ist. Daran sollten wir weiter fest halten. Insofern habe ich nicht die Befürchtung, dass sich das jetzt ab morgen ändern würde. Ich wüsste auch nicht, wer mich daran hindern sollte, im Seminar das zu erzählen, was ich glaube, erzählen zu wollen. Das wird man sicherlich immer können. Aber die Frage ist, wieso taucht diese Tendenz plötzlich auf, dass wir uns an diesen anderen Instituten, an diesen anderen Weiterbildungsordnungen orientieren wollen.

ML: Aber was meinst du damit: «Wir orientieren uns an den anderen?» Meinst du etwas Inhaltliches?

OK: Zum Beispiel die Aufteilung in Vorklinik und Klinik. Es wurde an der TV explizit gesagt, als das neue Modell vorgestellt wurde, dass man sich an den Weiterbildungsmodellen, wie sie bei den anderen Instituten bereits existieren, orientiert hätte. Dazu gehören auch die Interviews, die vorgesehen sind, oder die Einführung eines Status von Dozenten oder von Lehranalytikern ...

ML: Das sind viele Vermischungen! Ich gebe dir in einem Punkt recht, was die Aufteilung in klinisch und vorklinisch (oder umgekehrt) anbetrifft: die ist formal, die ist nicht gefordert, das ist eine «Eigenleistung» des PSZ ...

OK: Eine imitative Eigenleistung!

ML: Eigenproduktion ... Ich denke, dass man mit dieser Weiterbildung versucht, verschiedene Anliegen unter einen Hut zu bringen. Man verpackt da Sachen hinein, die nicht hinein gehören, die plötzlich reguliert werden. Zum Beispiel die hochfrequente Analyse. Niemals war im PSZ die Frequenz einer Analyse irgendwo festgelegt, geregelt, niedergeschrieben. Jetzt kommt das mit der PsychotherapieWeiterbildung; es ist keine Forderung der Charta, dass die Frequenz festgelegt wird. Auch das ist ein Produkt des PSZ, dass plötzlich hochfrequent dort drin steht.

OK: Das steht noch nicht drin, das ist ein Punkt, der ausgeklammert wurde. 
ML: Nein, das steht noch nicht drin. Aber es steht auf dem Flyer. Auf dem Flyer steht hochfrequente Analyse.

PP: Warum? Hat das etwa mit der Vertretung der psychoanalytischen Ideale zu tun? Es ist doch gerade umgekehrt! Für eine Therapieausbildung braucht man keine hochfrequente Analyse, wozu denn? Eine hochfrequente Analyse braucht man, wenn man Analysen machen will.

ML: Ich sage ja, dass sich da verschiedene Anliegen vermischen!

PP: Das widerspricht deiner These, dass man das trennen kann. Die therapeutischen Erfordernisse geraten in die Analyse und die analytischen in die Therapie und zwar in eine Therapie, die wir ursprünglich nicht gemeint haben! Ich möchte die Frage doch noch beantworten, ob das Abnehmen der analytischen Inhalte daran liege, dass man diese Therapieausbildung einführt. Das ist eine Frage wie die, ob es an der Gewalt in den Medien liege, dass die Gesellschaft gewaltsamer werde oder umgekehrt, ob die Medien deshalb mehr Gewalt zeigen, weil die Gesellschaft mehr Gewaltpotential hat. Das kann man nicht sagen. Das gehört zusammen.

SvA: Man kann es nicht beantworten. Sagen kann man es schon.

PP: Aber es ist doch klar, dass das im Gleichschritt läuft. In dem Masse, wie diese ausser-analytischen Standards wichtiger und eben auch freiwillig einbezogen werden, in dem Masse werden die analytischen weniger Gesprächsthema. Auch in der Analyse, das ist ganz klar. Aber man kann nicht sagen, das Eine bedinge das Andere, es ist die gleiche Tendenz.

Journal: Die Frage ist doch, wie soll man analytisch orientierte Psychotherapie vermitteln, wenn man keine psychoanalytische Grundlage hat? Natürlich kann man Psychotherapie auf verschiedenste Arten wirksam gestalten, das hat auch schon Freud gesagt, aber das ist nicht der Punkt. Sondern: wenn man an der psychoanalytisch orientierten Psychotherapie festhalten will, muss man doch die Psychoanalyse vermitteln!

ML: Aber weshalb muss man das niederschreiben und regeln?Weshalb können wir uns nicht darauf verlassen, dass es so bleibt, wie bis anhin (ich denke, es sei so gewesen), dass wir alle, wenn wir von Analyse in der Ausbildung zur Psychoanalytikerin 
gesprochen haben, an eine hochfrequente Analyse gedacht haben? Können wir im PSZ nicht mehr davon ausgehen, dass es ein Selbstverständnis ist? Das gilt auch für die Psychotherapie-Weiterbildung. Man könnte ja auch vom Selbstverständnis ausgehen, dass wer Psychotherapie betreiben will und diese Weiterbildung macht, eine hochfrequentige Analyse braucht. Aber das muss ja nicht niedergeschrieben werden, das hat doch etwas mit dem Selbstverständnis zu tun. Das meine ich, das schwindet.

OK: Dass wir das früher nicht so ausformuliert haben - hochfrequent, also drei oder vier Stunden -, hängt auch damit zusammen, dass es auch am Seminar Positionen gab, zum Beispiel von René Pomeranz, der ernsthaft die Meinung vertrat, er mache Analysen mit einer Stunde. Das sei keine Therapie, er mache Analyse mit einer Stunde. Man hat diese Fragen offen gelassen, nicht etwa weil man die Frage der Analyse für unwichtig erachtet hätte, sondern weil man das Spektrum dessen, was Analyse ist, offen halten wollte. Das ist ein wesentlicher Unterschied.

Ich halte es auch für ganz wichtig für die Psychotherapie-Ausbildung, dass diese Selbsterfahrung, die da jetzt gefordert ist, dass das eine psychoanalytische Selbsterfahrung ist. Das halte ich für unabdingbar. Aber, das muss nicht gleichbedeutend sein, dass wir schon von Anfang an fordern müssten, diese psychoanalytische Selbsterfahrung müsse wöchentlich drei oder vier Stunden betragen, in dem Sinn hochfrequent sein. Es gibt durchaus Fälle, wo man auch mit einer Stunde Analysen machen kann, mal ganz abgesehen davon, dass wir viele Situationen haben, wo jemand mit einer oder mit zwei Stunden anfängt und sich das dann sozusagen zu einer hochfrequenten Analyse ausweitet.

ML: Aber Weiterbildung und Klienten - das finde ich noch einen Unterschied!

OK: Nein, das finde ich jetzt keinen Unterschied.

PP: Aber das ist doch alles der Gipfel der Heuchelei! Warum kommt man jetzt plötzlich mit dieser Hochfrequenz? Nur weil das Patienten bringt, weil das Selbstversorgung ist! Ich sehe keinen anderen Grund. Der beste andere Grund, den man noch anführen könnte, wäre, dass man Formalitäten zu Hilfe nimmt, weil man den psychoanalytischen Geist nicht festhalten kann. Das war ja schon immer die Geschichte der IPA, oder nicht? Diese unsinnigen «minimal standards», wo man irgendwelche Formalitäten für die Sache selbst nimmt. Es ist eine Tatsache heute, dass kaum noch jemand hochfrequente Analysen macht! Ich kann praktisch 
niemanden mehr dazu bewegen, vier Stunden zu machen. Warum ist das so? Man kann sagen, die Arbeitsverhältnisse hätten sich geändert, es sei strenger geworden usw., aber es stimmt nicht. Zur Zeit, da wir angefangen haben (in den 70er Jahren), gab es viele Leute, die wollten vier Stunden Analyse haben und deshalb konnten sie das auch. Und heute gibt es niemanden mehr, der das will, und da muss man die Leute überzeugen, aber das geht gar nicht, weil die sagen, die anderen machen das auch nicht.

SvA: Woran liegt das, glaubst du?

PP: An diesem Zeitgeist, der von Effizienz trieft, von irgendwelchen Zielen, die erreicht werden sollen ... das Absurdeste ist zum Beispiel, dass jemand anruft und sagt, er möchte eine Analyse. Und dann kommt er und sagt, er mache die Ausbildung bei Frau Boothe an der Universität und dort müsse man eine hochfrequente Analyse, mindestens dreihundert Stunden machen. Jetzt wollen die in allem Ernst 300 Stunden hochfrequente Analyse und dann brechen sie ab. Nur für ihre Ausbildung! Das ist doch absurd!

SvA: Ist dir das passiert, dass die abbrechen? Hast du das erlebt?

PP: Nein, ich habe solche Klienten gar nicht genommen.

SvA: Ich hab auch solche Leute, aber ich habe noch niemanden erlebt, der nach 300 Stunden abgebrochen hat. Aber wenn du das jetzt so sagst:Was ist der Unterschied zwischen demjenigen, der zuerst eine Stunde kommt und sich dann verführen lässt oder sich so entwickelt, dass er drei Stunden kommen will auf der einen Seite und jene, die aus Boothe-Gründen drei Stunden kommen und dann abbrechen auf der anderen Seite?

PP: Ich weiss das von mehreren Kollegen und ich kenne das auch aus Deutschland.

SvA: Das ist etwas anderes, in Deutschland ist das wegen der Krankenkasse so.

PP: Aber das ist doch genau dasselbe. 
SvA: Ich finde nicht. Es ist ein Unterschied, ob jemand eine Ausbildung macht oder als Klient kommt.

PP: Entschuldigung, aber dieses Gerede von der Verführung, das kann ich nicht mehr hören, das ist ein derartig alter Hut, die Leute zu verführen, die Leute - man kann die Leute nur zu dem verführen, wozu sie verführt werden wollen.

SvA: Dann nimmst du ein anderes Wort. Es entwickelt sich doch ein Prozess. Der bricht nicht nach 300 Stunden ab, Peter.

PP: Nein, aber es ist auch nicht so, dass die Leute, die nur zwei Stunden kommen, keine Analysen machen.

SvA: Ist ja absurd. Nein!

PP: Und deshalb ist diese Hochfrequenz ein Fetisch. Die Hochfrequenz hat nur eine Bedeutung, nämlich dass es sich erfahrungsgemäss gezeigt hat, dass es so am besten geht. Es definiert nichts, gar nichts. Wenn man im Urwald ist und nur alle fünf Wochen mit dem Helikopter kommen kann, macht man eine Analyse in der Hängematte mit einer Stunde oder zwei Stunden alle Monate. Das geht auch, es geht einfach nicht so gut. Und jetzt sind dieVerhältnisse so, dass sich die Menschen in aller Regel nicht auf diesen Deal einlassen, aber deshalb kann man trotzdem Analysen machen. Man muss sie gar nicht erst zu vier Stunden verführen. Aber man lügt sich in die Tasche, wenn man meint, man könne gewisse Formalien hinüber nehmen und habe damit etwas von der Psychoanalyse gerettet. Die psychoanalytische Haltung ist etwas, was man im Kopf hat, das ist der Geist der Sache.

SvA: Ich möchte darauf zurückkommen, was mit unserem Seminar passiert. Abgesehen, soweit man das kann, von der Weiterbildung. Es hat mir gefallen, was am letzten Dozenten-Abend gesagt wurde, dass man die ökonomischen Verhältnisse berücksichtigen muss, wenn man jemanden in psychoanalytischer Ausbildung bei uns am Seminar hat. Es stimmt, es ist teuer, und ich bin damit sehr einverstanden, dass wenn man dann darauf besteht, dass jemand viermal in derWoche 140 Franken bezahlt, dass das kaum jemand kann. Es geht mir wirklich nicht darum, dass man das festschreibt. Ich kenne selber viele Prozesse, die analytisch verlaufen sind mit einer Stunde pro Woche während andere drei oder vier Stunden gekommen sind, und es war wirklich beinhart, und es hat nicht soviel herausgeschaut, wie ich mir 
erhofft hatte. Ich hänge nicht an diesen drei oder vier Stunden. Aber du hast vorhin deutlich gesagt, dass es sich gezeigt hat, dass das doch - wie hast du das gesagt?

PP: Es geht am besten so.

SvA: Es geht am besten so. Das können wir doch weiterhin vertreten und einen Modus finden, es anzubieten. Das müssen wir publik machen und uns nicht dauernd die Köpfe einschlagen wegen dieser Weiterbildung. Wir müssen schauen, dass wir wieder mehr Luft bekommen in dieses Feuer, damit es auch wieder besser brennt am PSZ. Dass wir darüber wieder sprechen, dass es am besten so geht oder sehr viel besser so geht, und eben doch eine tolle Erfahrung ist! Dass man drei oder vier Stunden in Analyse gehen kann. Ich habe selbst viele Jahre mit vier Stunden erlebt, und ich muss sagen, es war eine super Erfahrung. Das ist es, was wir besetzen müssten! Du kannst das Wort «verführen» streichen. Das müssten wir affektiv besetzen, und da müssten wir uns halt dazu durchringen, wenn wir das wirklich pflegen wollen, dass wir Leuten anbieten, für weniger Geld zu kommen. Ich mache das zum Teil schon. Wenn ich sehe, die können das nicht wirklich bezahlen. Auch mit Supervisionen, finde ich, muss man mit dem Preis runter. So können wir das pflegen, was wir inhaltlich möchten!

PP: Völlig einverstanden.

SvA: Das ist mir wichtig, das ist mir einfach wichtig ...

OK: Bin ich auch vollkommen deiner Meinung. Ich handhabe das auch so, dass ich dann zum Beispiel mit dem Preis runtergehe. Aber das ist eine individuelle Entscheidung, die jeder einzelne trifft. Das, worum es hier geht ...

SvA: Aber diese Diskussion muss doch öffentlich an unserem Seminar geführt werden, wie wir das zu handhaben gedenken.

ML: Man könnte vielleicht sagen, das wäre die Alternative: Statt dass wir regeln, und hochfrequente Analyse festschreiben ...

OK: Ich verstehe jetzt, ehrlich gesagt, den Affekt nicht! Ich habe euch doch daran erinnert, dass genau das eben noch nicht festgelegt sei. An der letzten TV ist dieser 
Punkt als einziger ausgeklammert worden, weil man sagte, darüber müsse man reden. Und du tust jetzt so, als ob wir das jetzt nicht ...

SvA: Ja, Olaf, das ist ein Missverständnis. Du hast gesagt «Ich mache das schon lange so.» Das glaube ich dir auch, es geht darum, dass es publik gemacht wird. Nicht, dass man es fest schreibt. Und ich rede jetzt nicht von der Weiterbildung. Ich rede von der Psychoanalyse, dass die Möglichkeit am PSZ besteht, dass sich jemand bei verschiedenen Analytikern umsieht, und dass wir auch darüber sprechen, günstiger zu arbeiten oder ein paar Stunden die Woche frei zu halten für solche Analysanden, die wirklich vier Stunden kommen wollen. Ich meine nur, dass es auch vermittelt werden soll.

PP: Wenn ich sage «es geht so am besten», dann meine ich, es geht so am leichtesten, einen analytischen Prozess in Gang zu bringen und aufrecht zu erhalten. Man kann das aber nicht dem Ökonomischen gleichsetzen. Ich bin absolut überzeugt, dass es Analysen gibt mit vier Wochenstunden, die geben am Schluss mehr Stunden, als solche mit zwei. Und das ist heute aber das Kriterium! Die Effizienz! Wenn einer kommt und sagt, ja wenn man es doch auch mit einer oder zwei machen kann, wieso dann mit vier? Weil es besser geht, aber nicht weil es kürzer geht oder effizienter.

SvA: Das glaube ich nicht.

PP: Doch, das ist so.

SvA: Ich glaube nicht, dass jemand, der eine Psychoanalyse haben will, auf Effizienz achtet. Es ist nicht jeder so beschränkt. Es gibt Leute, die eine Analyse wollen, um diese Erfahrung zu machen, und nicht weil es effizient sein soll.

PP: Ich habe gerade in letzter Zeit erlebt, dass es solche Leute hat, aber von denen gibt es eben weniger. Das ist der Zeitgeist.

SvA: Das stimmt. Weniger, aber nicht keine.

PP: Und das hängt nicht einfach davon ab, was wir tun, das ist eine grosse Maschinerie, in der das so läuft. Da sollten wir uns ausklinken und hätten eh und 
je vertreten sollen, dass wir etwas anderes anzubieten haben und das hochhalten. Aber stattdessen schleichen wir ...

SvA: Können wir jetzt das nicht mehr anbieten und hochhalten?

PP: Wir können das schon. Ich hoffe das wir es noch tun, aber es ist nicht sehr wahrscheinlich.

SvA: Aber genau das müssen wir tun!

Journal: Die Frage ist, ob die Institutionalisierungsschritte, die jetzt gemacht werden, um die staatliche Anerkennung zu bekommen, das noch erlauben, was ihr alle möchtet, nämlich die Psychoanalyse stärker zu besetzen. Oder ob diese Institutionalisierungsschritte derart sind, dass sich das Seminar in eine neue Richtung entwickelt. In eine bürokratische Richtung.

PP: Es ist doch klar, dass zum Beispiel eine hochfrequente Analyse sehr bald als therapeutischer Kunstfehler bezeichnet werden wird, weil es nicht angeht, dass man die Leute zu einer ineffizienten Methode verführt! Und dann werden wir gezwungen sein, das völlig zu trennen. Wir können heute ja noch sagen, eine Psychoanalyse sei auch eine Therapie, aber wenn wir erst einmal so weit gegangen sind mit der Akzeptanz all dieser Regeln, dann schlägt das plötzlich zurück und dann heisst es: Eine Psychoanalyse ist keine Therapie, hat gar nichts damit zu tun. Dann muss man sie völlig davon trennen.

ML: Aber das ist ja gar nicht, was gefordert wird. Von aussen wird keine hochfrequente Analyse gefordert.

PP: Nein, im Gegenteil.

ML: Das ist unser eigener Anspruch.

PP: Aber ich will nur sagen, dass dann diese Kriterien von aussen unter Druck geraten werden, die eigentlichen, die wir jetzt für analytisch halten.

Journal: Ihr seid euch im Grossen und Ganzen einig darüber, dass man die Frage der hochfrequenten versus der niederfrequenten Analyse nicht allzu sehr 
betonen sollte, und dass mit beiden Settings Analysen möglich sind. Ich möchte noch einmal auf den Anfang der Diskussion zurückkommen, nämlich auf die Institutionalisierungsschritte, die anstehen, damit die Weiterbildung in analytisch orientierter Psychotherapie möglich und staatlich anerkannt werden kann. Einer dieser Schritte wird wohl sein müssen, dass unsere einfache Gesellschaft zugunsten einer Vereinsstruktur aufgegeben wird. Was denkt ihr darüber?

ML: Ich finde es tatsächlich schade, wenn das aufgegeben wird, auch wenn es verschiedene Stimmen gibt, weshalb das PSZ organisatorisch eine einfache Gesellschaft ist. Die einen sagen, das sei gar nicht freiwillig so gewählt worden, sondern es sei ein Zufallsentscheid gewesen, dass man sich so vereint habe. Ich finde es trotzdem ganz wichtig, weil in der einfachen Gesellschaft jeder für das Ganze verantwortlich ist. Mit der eigenen Person. Mit dem Verein ist es anders, da gibt es dann die juristische Person des Vereins, es ist eine Delegation sozusagen. Und das finde ich von der Befindlichkeit her doch ein grosser Unterschied. Deshalb sperre ich mich auch so dagegen, dass man das ganze PSZ jetzt zum Verein macht. Ich bin der Meinung, dass man den Verein dort macht, wo er notwendig ist, und nicht das Ganze in einen Verein umwandelt.

OK: Mir geht's ähnlich wie Monika, ich würde das auch bedauern und zwar vor allem deswegen, weil ich glaube, dass in dieser einfachen Gesellschaft genau diese Art von gegenseitiger und gemeinsamerVerbindlichkeit gegenüber dem PSZ einerseits und gegenüber dem, was wir unter Psychoanalyse verstehen, anderseits zum Ausdruck kommt. Insofern ist mir diese einfache Gesellschaft immer auch lieb gewesen; gleichzeitig finde ich aber auch, muss man sagen, dass wir de facto wie ein Verein funktionieren. Wir haben Statuten und Geschäftsordnungen, z. B. wie man an der TV abstimmt usw. Von daher glaube ich nicht, dass sich am Betrieb des Seminars etwas Wesentliches ändern würde, wenn wir einen Verein gründen. Die Idee, die in dieser einfachen Gesellschaft steckt, ist mir auch wichtig, aber die hängt auch nicht nur an der einfachen Gesellschaft. Es kommt auf die Art und Weise an, wie wir zusammen dieses Seminar betreiben. Für mich ist das kein Entweder-Oder.

SvA: Du würdest das Ganze als Verein sehen? Was sagst du zu der Trennung zwischen Weiterbildung und PSZ?

OK: Ich bin ganz und gar nicht für diese Trennung. 
PP: Ich hänge auch an dieser einfachen Gesellschaft - aus symbolischen Gründen. Ich bin nicht so überzeugt, ob derVerein nicht tatsächlich eine grosse Veränderung bringt. Wir funktionieren zwar wie ein Verein, aber wir sind eben doch keiner. Vieles von dem, was ja auch ärgerlich ist, hat damit zu tun. Sylvia hat vorhin dieses Stagnieren beklagt. Aber das finde ich auch eine Qualität. Wenn in einem Verein ein paar zügige Leute an die Spitze kommen, dann passiert etwas. Dann wird geändert, dann wird einmal ausgemistet und dann werden die faulen Eier weggeworfen und dann kommt ein neuerWind rein usw. Und dann gibt es sicher sehr viel mehr Dynamik, aber ich glaube nicht, dass das gut ist. Ich glaube, dass die einfache Gesellschaft etwas in meinem Sinne sehr positiv Konservatives an sich hat. Dass es eben sehr schwierig ist, die Dinge einfach umzustürzen. Und deshalb bin ich auch gegen den Verein. Ich habe eine fast körperliche Abneigung gegen Vereinsmeierei. Und es ist halt so, dass in einem Verein die Strukturen, die Mittel, sehr schnell wichtiger werden, als die angestrebten Ziele, als derVereinszweck. Das ist ja etwas sehr Typisches, dieses Selbstzweckhafte, die sich aufblähende Bürokratie, die dann schlussendlich völlig in Vergessenheit geraten lässt, worum es eigentlich geht.

OK: Im Zusammenhang mit der Gefahr der Bürokratisierung wäre ich sehr dagegen, dass man jemanden als eine Art Schulleiter anstellt, einen so genannten Fachmann, der die administrative Arbeit zu 20\% oder zu wie viel \% übernehmen würde - das ist ja auch ein Vorschlag, der gemacht wurde. Ich glaube, das wäre genau der Anfang einer solchen bürokratischen Entwicklung, wenn man jemandem die Administration delegiert. Der würde sich da hinein beissen, und es ist absolut voraussehbar, dass sich ziemlich schnell heraus stellen würde, dass es nicht nur 20 sondern 30 und $40 \%$ brauchen würde, und dass genau dieser Bereich der Bürokratie, für den er angestellt worden wäre, eine immer grössere Bedeutung bekommen würde. Deswegen wäre ich sehr dagegen, dass man das macht. Wir haben auch in unserer Öffentlichkeitsarbeits-Gruppe sehr viel zu tun, aber es ist machbar. Damit behalten wir die Dinge in unserer Hand. Das ist auch ein Teil dessen, was in dieser einfachen Gesellschaft zum Ausdruck gekommen ist.

ML: Ich bin mit deinem vorherigen Votum nicht einverstanden, Olaf. Ich denke, dass eine Vereinsgründung sehr wohl etwas ändert, auch wenn wir jetzt schon Vereinsstrukturen haben. Tatsächlich sind wir kein Verein. Ich denke nicht, dass wir das einfach austauschen können und sagen: «Ja, es kommt eigentlich gar nicht so drauf an.» Denn das Bewusstsein darüber, dass wir eben kein Verein sind, ist von grosser Bedeutung. Von da her verstehe ich es nicht, weshalb du so vehement gegen 
diese Trennung bist. Das, was einen Verein braucht, das ist die PsychotherapieWeiterbildung, die soll ihn doch bekommen. Weshalb muss sich denn das ganze PSZ dem unterordnen?

OK: Darüber haben wir ja vorhin gesprochen. Das hat etwas mit dieser Trennung zu tun, von der ich glaube, dass man sie nicht machen kann und nicht machen sollte. Natürlich soll sich das PSZ nicht von der Weiterbildung dominieren lassen. Aber ich bin auch nicht der Meinung, dass es sinnvoll ist, die Weiterbildung in einen Verein auszugrenzen und sich dann sozusagen der Illusion hinzugeben, wir hätten damit nichts mehr zu tun. Weil derVerein, das sind ja auch wir, wir müssen die Arbeit ja genau dort auch machen, das ist ja nicht irgendjemand anderes.

Journal: Ein weiterer wichtiger Punkt ist, dass staatlich von einem anerkannten Seminaroder Instituterwartetwird,dassaucheineQualifizierungder «KandidatInnen», vorgenommen wird. In derTV wurde beschlossen, dass ein Ausschuss gebildet wird, welcher die AnalytikerInnen in Ausbildung begleitet und an verschiedenen Punkten des Curriculums mit ihnen Qualifikationsgespräche durchführt. Muss man das nicht als einen hierarchischen Schritt anschauen, dem ebenfalls die Gefahr der Bürokratisierung anhaftet?

ML: Was im Curriculum festgelegt wurde, ist genau das, was verlangt wird. Es braucht eine so genannte Prozesskontrolle. Das wurde auf der Ebene eines Standortgesprächs installiert, nicht einer Qualifikation. Die Qualifikation ist nicht gefordert. Die steht im Curriculum so auch nicht drin. Und in dem Sinn ist es eigentlich nicht eine Hierarchisierung oder eine Institutionalisierung. Wie das dann gehandhabt wird, das ist eine andere Frage.

Journal: Die Intention wäre eine Begleitung. Ohne Qualifikation.

ML: Ja, eine Begleitung. Ohne Qualifikation.

OK: So, meine ich, haben wir auch abgestimmt, glaube ich.

ML: So haben wir abgestimmt ... 
OK: Also durchaus auch auf deinen, aber auch auf meinen Antrag hin, dass das eben expliziert wird, dass das keine irgendwie qualifizierende Funktion hat (Zustimmung aller).

Journal: Dasstimmt, daswardie Intention, die so abgestimmt worden ist. Ich frage trotzdem: Wie werden sich solche Weichenstellungen in der Zukunft entwickeln? Seid ihr der Meinung, dass das Seminarkollektiv freiheitlich genug ist, um allfällige Machtbedürfnisse von neu zu bildenden Ausschüssen Paroli zu bieten? Oder muss man befürchten, dass sich eine unbewusst ablaufende institutionelle Entwicklung ergibt, die doch zu einer Hierarchisierung und Bürokratisierung führen kann?

SvA: Ich glaube schon, dass diese Gefahr besteht, das ist keine Frage. Aber für die Weiterbildung muss es ja einen Verein geben - angenommen, die meisten denken so wie wir, und es wird beschlossen, dass wir eine einfache Gesellschaft bleiben, dann würde die Weiterbildung doch flach fallen? Zum anderen glaube ich, dass wir die Möglichkeit haben, einer Hierarchisierung entgegen zu wirken - das hängt doch sehr davon ab, wie wir uns dagegen einsetzen. Dass man zum Beispiel an die TVs geht. Wir müssen diese Instrumente benutzen, die wir haben und die am PSZ nach wie vor gelten. Man kann nur versuchen, mit unseren Methoden daran zu bleiben, dass man nicht insofern eine Spaltung installiert, dass gewisse Leute nicht mehr kommen oder dass man das Feld einfach räumt. Aber das war schon immer so. Das ist angelegt in dieser Art von Basisdemokratie, wie wir sie haben, dass es darauf ankommt, wer teilnimmt, wer mitdiskutiert und wer sich einlässt. Das ist ja auch nicht neu, wir müssen es einfach weiterführen.

OK: Ich bin absolut deiner Meinung. Ich sehe aber auch die Gefahr der Institutionalisierung. Es werden bereits Regelungen vorgesehen, die über das hinausgehen, was eigentlich gefordert ist. Das ist Ausdruck einer Tendenz, die es am Seminar schon immer gegeben hat. Es hat immer auch Wünsche und Forderungen gegeben, man müsse solche Hierarchisierungen irgendwie einführen. Die bekommen heute mehr Gewicht.

PP: Freud wurde einmal gefragt, wieso er, wenn sein Begriff von Sexualität gar nicht mehr identisch sei mit der biologischen Sexualität und viel näher beim allgemeinen Begriff der Liebe stehe, warum er sie dann nicht einfach so nennen würde. Er antwortete «Weil man zuerst in den Wörtern nachgibt und dann in der Sache.» Das ist immer so. Und wenn es jetzt «Standortgespräche» heisst, stellen sich mir 
die Haare zu Berge, weil ich das aus der Wirtschaft kenne, aus den Institutionen. Es sind lauter Euphemismen, aber darunter wird die Schraube immer mehr angezogen. Ich weiss nicht, ob ihr das einmal gelesen habt, im Tagesanzeiger, wie ein Personalmanager einem Mitarbeiter (ich glaube einem 55-jährigen Mann) seine Stelle kündigt und es fertig bringt, das so zu machen, dass der das nachher noch eine gute Sache findet ... Es geht um Selektion und um nichts anderes. Sonst könnte man es bleiben lassen.

ML: Nein, wir können es nicht bleiben lassen. Wenn wir diese Weiterbildung machen wollen, dann können wir ...

PP: Aber Monika, das ist jetzt ständig diese Erpressung! Und wenn es am Schluss heisst, wir müssen die Todesstrafe einführen, wenn wir diese Weiterbildung machen wollen, dann führen wir eben die Todesstrafe ein? Aber ich will diese Weiterbildung gar nicht machen, weil sie bedeutet ja nichts anderes, als dass wir lauter solche Zwänge übernehmen müssen, die wir gar nicht wollen!

SvA: Aber das ist jetzt beschlossen!

PP: Ihr könnt mir sagen, was ihr wollt, aber ich bin dagegen.

SvA: Es ist nicht mehr rückgängig zu machen.

Journal: Ist es nicht so, dass diese Verschulungstendenz weitgehend auch eine Generationenfrage ist? Dass ein grosser Teil der jüngeren KollegInnen solche Institutionalisierungsschritte und auch Prüfungen wünschen?

OK: Das glaube ich nicht, die ganzen Vorschläge kommen doch nicht von der jüngeren Generation, die kommen aus unserer eigenen Generation! Es sind die, die immer schon Lehrer werden wollten und Dozenten am Seminar, darum geht's!

SvA: Ich glaube dagegen, dass das mit den Jungen stimmt. Ich denke, die werden jetzt so sozialisiert im Bildungswesen. Alle Universitätslehrgänge haben sich geändert, alles wurde strukturiert, da ist ein riesiger Institutionalisierungsschritt im Gang. Sie sind sich das eher gewöhnt, als wir das waren. 
OK: Aber das war auch immer schon so. Ausserdem haben wir natürlich weniger Junge, also weniger Interessenten am Seminar. Aber wir haben weniger, weil überhaupt die Psychoanalyse weniger hat. In Deutschland, in Frankfurt gibt es zum Beispiel kaum noch Kandidaten! Dass der Zulauf auch bei uns abgenommen hat, liegt nicht an unserer Art der Ausbildung oder Weiterbildung, sondern es ist ein allgemeiner Trend. Aber die Gefahr ist ständig, dass wir meinen, die kommen nicht, weil wir es nicht richtig machen. Darin kommt ständig eine Selbst-Entwertung und eine Entwertung des Seminars zum Ausdruck, die vollkommen abwegig ist.

PP: In den 70er Jahren waren die Jungen die Träger einer Ideologie, die ganz gegen Prüfungen usw. eingestellt war. Heute gehen sie mit dem Zeitgeist. Wir sind nicht gegen die Jungen, wenn wir gegen den Zeitgeist sind. Das sind zwei verschiedene Dinge.

OK: Was ich auch glaube, ist, dass die Jungen in Bezug auf diese neuen Regelungen auch gleichzeitig sehr viel flexibler sind. Der Umgang mit den Hierarchien ist irgendwie auch ein ganz anderer geworden. Ausserdem haben wir nicht weniger Junge am Seminar als beispielsweise das Freud-Institut oder die Boothe, die sind ja genauso in der Krise mit dem Nachwuchs.

SvA: Ich fühle mich angesprochen, weil ich immer wieder die Frage aufgebracht habe: «Wo sind die Jungen?» Aber ich habe nie danach geschrieen, wo sind die Jungen am Seminar, in den Ausbildungen. Da wäre ich völlig missverstanden worden. Ich habe gefragt, wo sind die Jungen, wenn es darum geht, unsere TV abzuhalten. Wie sie sich an der Organisation des Seminars beteiligen. Da habe ich gesagt, wo sind die Jungen. Ich habe mich gefragt (und das geht wirklich unsere Generation was an), wie machen wir das, dass wir neben dieserWeiterbildung unser psychoanalytisches Seminar tradieren, wie machen wir das, dass die Jungen, die Neuen, kommen, daran teilnehmen, das weiter tragen oder das weiter entwickeln. Wie bringen wir das fertig, dass unsere Kultur nicht einfach verschwindet. Zum Beispiel habe ich gehört, dass Leute, die in einem Seminar waren, in der Kneipe die Analytikerin, die das Seminar gegeben hat, völlig erstaunt fragten: «Was, ihr habt keinen Lohn?» Ich meine solche Dinge, oder, dass die Leute nicht wissen, wie es hier funktioniert, aber die Weiterbildung machen, das finde ich krass. Deswegen bin ich mal mit dieser vielleicht blöden Idee gekommen, man sollte das im Curriculum oder im Flyer festlegen, dass die Leute, die am PSZ eingeschrieben sind und hier Weiterbildung machen, auch an TVs teilnehmen müssen. Ich weiss, es wäre heikel, so was fest- 
zuschreiben. Ausdrücken wollte ich damit, dass wir stärker in Erscheinung treten müssen mit dem, was wir vertreten.

PP: Aber du würdest doch auch sagen, früher, also in dieser Zeit, in die wir jetzt immer nostalgisch zurückblicken, sind wir doch an die TV nicht deshalb gegangen, weil wir mussten, sondern weil wir gerne hingingen, es war interessant.

SvA: Weil wir wussten, dass das PSZ so funktioniert. Wissen das die Leute noch?

PP: Von Blarer hat doch dieses Wort geprägt «der Weg ist das Ziel», das heisst, eine Analyse ist doch ein Weg, der einen Wert in sich hat und nicht primär als Ziel. Heute geht es aber immer um Zielvereinbarungen, Pragmatik, Effizienz. Wenn im vorherrschenden Zeitgeist alle diese Werte so hoch veranschlagt werden, kann die Psychoanalyse, die sich um das alles eigentlich nicht kümmert, keinen hohen Stellenwert haben. Man sollte nicht versuchen, mit Kosmetik das zu umgehen, in dem man es anpasst, markttechnisch unter falschem Etikett verkauft. Man sollte im Gegenteil laut und deutlich sagen, was Psychoanalyse ist, weil es nämlich - und davon bin ich überzeugt - auch unter diesem Zeitgeist ganz viele Leute gibt, die damit nicht übereinstimmen, die auf so etwas ganz anderes eben scharf sind. Aber wenn man das alles versteckt hinterWeiterbildung und hinter Psychotherapie und weiss was, dann muss man sich nicht wundern, wenn niemand mehr kommt.

SvA: Du sprichst vom Selben, wie ich vorhin, wir müssen uns nicht verstecken und unsere Ideale unter den Teppich wischen, sondern das deklarieren und im Seminar kultivieren. Und es gibt ganz sicher Leute, die schon lange die Nase voll haben von dieser Pseudo-Effizienz, da bin ich überzeugt. Aber das muss sichtbar sein und bleiben an unserem Seminar. Dafür müssen wir uns einsetzen.

Journal: Ich habe noch eine Frage nach der Selbstautorisierung, die immer ein Qualitätszeichen unseres Seminars gewesen ist. Ob mit diesem neuen Modell der Begleitung,dernichtwirklich ausgesprochenen Qualifikation, die Selbstautorisierung noch einen Stellenwert hat?

ML: Bei der Weiterbildung in Psychotherapie gibt es keine Selbstautorisierung. Wir verleihen ein Zertifikat! Das ist eine verschulte Weiterbildung, die Vorgaben hat, die wir umsetzen, und die wir mit einem Zertifikat beenden. 
PP: Aber die Frage ist doch, ob so etwas nicht eine Rückwirkung hat auf die Institution, die das anbietet. Kann man so verschiedene Dinge unter einem Dach verbinden?

OK: Ich war immer gegen Zertifikate, aber ich glaube, selbst wenn es solche Zertifikate gibt, kommt derjenige, der die zertifizierte Weiterbildung absolviert hat, nicht um diesen Punkt der Selbstautorisierung herum, weil er auch dann wird entscheiden müssen, ob er jetzt Patienten gegenüber tritt und sagt: «Ich bin jetzt der Klaus Dieter und ich mache Psychoanalyse.» Das ist ein Schritt, den er nach wie vor verantworten muss, und das ist auch nicht ein Schritt, den wir ihm mit dieser Zertifizierung abnehmen können (das sollten wir uns auch selber nicht vormachen!).

PP: Aber meinst du Psychoanalyse oder Psychotherapie? Du hast jetzt Psychoanalyse gesagt.

OK: Ich meine, das gilt auch für dieWeiterbildung in Psychotherapie, weil sie eine psychoanalytische Psychotherapie ist. Es gilt für die Psychoanalyse ohnehin ...

PP: Aber da befindest du dich in einemWunschdenken. Gemeint ist, dass man am PSZ ein Curriculum durchläuft und zertifiziert wird. Dann kann man hingehen und sagen: «Ich bin zertifiziert und als solcher trete ich den Leuten gegenüber.» Das ist ja gerade das Problem, dass man das in diesem Bereich überhaupt nicht wirklich kann. Das war ja unsere Weisheit. Man kann nicht mit einem Menschen in einen therapeutischen Prozess treten, nur weil die Universität gesagt hat, man könne das. Aber genau das übernehmen wir jetzt auf der Schiene der Psychotherapie und machen eine Spaltung zwischen Psychotherapie und Psychoanalyse. Ich glaube aber, dass wir erkennen müssen, dass das auch auf der Ebene der Psychotherapie nicht geht. Und wenn wir das so sehen, können wir doch eine solche PsychotherapieWeiterbildung unter unserem Dach gar nicht verantworten.

ML: Ich bin da noch viel pessimistischer. Ich denke, dass sich der grosse Teil, der zertifiziert worden ist, nachher Psychoanalytiker nennen wird.

OK: Ich bin derselben Meinung, dass das Zertifikat nicht genügt. Aber ich glaube, dass wir das gleichzeitig während derWeiterbildung den Leuten klar machen können (das ist auch einer dieser Widersprüche, die nicht einfach auflösbar sind). Sie 
haben zwar das Zertifikat, aber sie wissen nichts desto trotz, dass der Akt, sich irgendwie dann in eine Praxis reinzusetzen und Patienten zu empfangen, noch mit viel mehr verbunden ist.

PP: Aber kannst du dir tatsächlich vorstellen, dass einer mit dem Zertifikat in der Hand sagt: «Ja, aber innerlich sehe ich, ich bin eigentlich kein Psychoanalytiker, ich mache jetzt keine Praxis.»

OK: Nein, es geht ja nicht darum, dass er keine Praxis macht. Das ist nicht der Punkt. So meine ich es auch nicht, aber dass man wohl deutlich macht, dass dieser Akt, sich in eine Praxis reinzusetzen, sehr wohl (auch dann, wenn man ein Zertifikat hat) etwas mit einer Selbstautorisierung zu tun hat.

SvA: Wenn du sagst, das muss man den Leuten klar machen, dann ist ja die Frage (das ist eigentlich mein grössterWunsch), dass wir uns dessen bewusst werden, wie man das macht. Das kann man nicht einfach festschreiben. Da muss sich das PSZ noch weiterentwickeln. Wir müssen versuchen, uns nicht auffressen zu lassen von diesemWeiterbildungs-Koloss, das vielleicht gar kein Koloss, sondern nur ein Zwerg ist. Wenn wir so weiter sprechen am PSZ und das kultivieren, was wir tun, dann wird es doch einigen Leuten klar werden und sie werden davon angesteckt. Und die machen weiter, auch eine vierstündige Analyse. Ich glaube, es geht sowieso nur so. Wieso müssen wir uns so wahnsinnig um diese Reglementierung kümmern?

Journal: Als Arzt konnte ich schon ganz am Anfang meiner PsychoanalyseWeiterbildung Psychotherapien machen. Ich hatte da diese Lizenz (ich war als Arzt zertifiziert). Aber das hatte nichts zu tun mit meiner Selbstautorisierung, ich fühlte mich überhauptnichtals Analytiker.Meine Selbstautorisierung ist viel später gekommen, in einem Prozess. Und das wäre die Frage: Inwiefern dieseWeiterbildung, die beschlossen worden ist, nur in dem Sinne eine Zertifizierung impliziert, dass die Leute ihre Praxis eröffnen dürfen, sich deswegen aber noch lange nicht selbst autorisiert haben?

PP: Gut, aber das gilt ja seit eh und je für überhaupt alle Menschen. Ich meine, bevor ein Arzt einen weissen Kittel anzieht und eine eigene Praxis eröffnet, muss er einen Schritt der Selbstautorisierung machen, wie jeder, der etwas macht. Aber ich hatte immer den Eindruck, wir hätten das etwas schärfer gefasst. Wenn man es darauf reduziert, dass schlussendlich alles, was man macht, einen selbstautoritativen 
Schritt erfordert, dann kann niemand mehr dagegen sein, muss man aber auch nicht speziell dafür sein ...Nein, es geht nicht nur um den Schritt, alleine etwas zu tun, sondern es geht um die Identität, die man sich erworben hat. Also eine psychoanalytische Identität. Es kann einem niemand zum Analytiker oder zum Therapeuten autorisieren. Aber genau das wird in diesem Modell halt gemacht. Dort wird gesagt: «Ja, der Staat, die Charta, wir (so in dieser Reihenfolge), wir autorisieren jetzt.» Und das ist ein Bruch mit unserem Selbstverständnis.

OK: Das ist so. Aber wir befinden uns in einem Feld, um das wir nicht herum kommen. Da der Kanton Zürich eine Psychotherapie-Regelung eingeführt hat, die beinhaltet, dass man dann eine eigene Praxis aufmachen kann wenn man die Bedingungen erfüllt hat, dann ist es halt so. Das sind die äusseren Regelungen. Deswegen muss man umso mehr zum Ausdruck bringen, proklamieren und vertreten, dass das nicht genügt. Dass es bei dem, was wir tun, immer auch um dieses Moment, um diesen Akt von Selbstautorisierung geht. Und zwar auch dann - und dann in besonderer Weise -, wenn man nun ein solches Zertifikat besitzt.

Journal: Heisstdas,dassnachdemjemandsichdiesesZertifikatals PsychotherapeutIn erworben hat, es noch einen weiteren Schritt geben könnte und geben sollte, nämlich PsychoanalytikerIn zu werden?

ML: Ja, das würde es eigentlich heissen!

SvA: Ja, sicher.

ML: Es ist eigentlich dasselbe, wie es bei den Medizinern schon immer war. Die haben sich ihre Psychotherapie-Titel geholt und sind dann am Seminar verblieben und haben sich psychoanalytisch weiter gebildet.

OK: Ich würde es nicht nur auf diesen Unterschied Psychoanalyse und Psychotherapie reduzieren. Als Arzt hattest du die «licence to kill». Du konntest also Psychotherapien machen, aber du hast erst Jahre später sagen können: «Okay, jetzt mach ich auch Psychoanalysen.» Dieser Akt, von dem ich spreche, bezieht sich nicht nur darauf, sich psychoanalytisch weiterzubilden. Das gilt auch für die Psychotherapie selber, für den Punkt, wo man sagt: «Jetzt bin ich Psychotherapeut.» Und der fällt nicht damit zusammen, dass man die Praxis aufmacht. 
PP: Aber kommen wir da nicht in eine gefährliche Nähe zu modernen Priestern oder Theologen, die gesehen haben, dass alle Züge abgefahren sind und die, auch wenn die ganze Welt sagt: «Es gibt keinen Gott», weiterhin behaupten: Es gibt ihn, und jeder weiss das im Herzen und eigentlich sind auch die, die nicht in die Kirche gehen, Gotteskinder, und wenn sie ihre Arbeit gut machen, dann gehören sie auch zur grossen Gemeinde? Damit rettet man, was längst verloren ist, in dem man es begrifflich soweit öffnet, dass niemand mehr nicht dabei sein kann. Aber das war ja eigentlich nicht, was wir wollten.

OK: Ich sage ja auch nicht, dass es das ist, was wir machen wollten. Aber nichts desto trotz glaube ich, dass es ein wichtiger Punkt ist. Du ziehst ihn jetzt auf die Art und Weise ins Lächerliche. Ich kann darüber durchaus auch mit dir lachen, aber du weisst genauso auch, dass es nicht nur lächerlich ist, dass diese Priester vielleicht nicht nur unrecht haben, und, dass es einem ja häufig genug so geht und, dass es auch nicht einfach nur etwas von Grund auf Falsches ist. Das vertrittst du ja selber oft genug...

PP: Jaja ...

SvA: Und was heisst das, wenn du sagst, das ist längst schon verloren? Wieso sprichst du davon, dass die - ich habe dich wohl falsch verstanden - dass die Psychoanalyse oder das, was man damit verfolgt hat, längst schon verloren ist?

PP: Ich glaube, dass sie als eine Idee, die eine öffentliche Wirkung hat, die ein Ansehen hat - das ist verloren, ja.

SvA: Das glaubst du?

PP: Zurzeit. Ich glaube, dass das in einer Bewegung zu sehen ist. Ich glaube, dass das immer so ist, dass gewisse Dinge verloren gehen und dann wieder kommen. Sie bleiben irgendwo. Ich finde eben, der verkrampfte Versuch, sie am Leben zu erhalten, dort, wo sie eigentlich am wenigsten leben können, wo sie dann erstickt werden, dagegen bin ich. Ich finde, man muss akzeptieren, dass eine solche Idee irgendwie in den Untergrund abtauchen muss, weil es über Grund keinen Ort mehr dafür gibt, aber dann sollte man versuchen, sie dort zu kultivieren und sie nicht auf Teufel komm raus oben mit lauter Begriffsdehnungen und -verdrehungen dann doch am Leben zu erhalten. 
SvA: Aber glaubst du nach dieser Diskussion, die nicht nur hier geführt wurde, dass das PSZ kein Ort mehr für die Kultivierung der Psychoanalyse ist? Glaubst du, dass das PSZ diesen Ort, den du jetzt als «Untergrund» bezeichnet hast, wirklich nicht mehr darstellt und nicht mehr zur Verfügung stellen kann?

PP: Ich glaube, dass das PSZ ein Ort ist, wo Leute, die so etwas vertreten, noch immer toleriert werden, das auch tun können. Aber ich glaube nicht, dass das den Geist des PSZ heute insgesamt ausmacht.

OK: Das ist für mich auch wieder zu sehr schwarz/weiss gedacht. Ich glaube auch, dass die Psychoanalyse in ihrer gesellschaftlichen Bedeutung deutlich abgenommen hat, wenn man den Vergleich zieht zu vor 30 Jahren. Ob ihr Stellenwert allerdings gänzlich verloren ist, da wäre ich mir nicht so sicher. In Bezug darauf, wo man sich vor allem einsetzen soll, bin ich durchaus auch der Meinung, dass man es nicht mit aller Kraft in der Klinik versuchen muss. Das ist auch ein Grund, weshalb wir diesen Jubiläums-Preis anders positioniert haben. Ich glaube wirklich, dass die Psychoanalyse im Moment teilweise an anderen Orten wirklich besser überlebt, als in dieser klinischen Situation. Das bedeutet aber nicht gleichzeitig, dass ich es hier einfach gänzlich aufgebe, das hat damit gar nichts zu tun. Das macht meine Position vielleicht etwas klarer: Auch dieser Akt der Selbstautorisierung, an dem muss man festhalten, ganz gleich wie es läuft.

SvA: Ja, ich finde es keine Verkrampfung, wenn wir sagen: «Wir versuchen, uns damit auseinanderzusetzen am PSZ, das zu machen, was wir für richtig finden». Trotz dieser Auseinandersetzung, die uns weiss Gott schon genug beschäftigt hat. Ich meine die Psychotherapie-Weiterbildung.

PP: Nur noch eine letzte Frage:Wenn das alles so ist, wie ihr das sagt, wieso brauchen wir dann diese Weiterbildung? Es gibt ja tausend Weiterbildungen! Wieso können Leute, die beim Freud-Institut das machen, oder bei den Verhaltenstherapeuten, wenn sie die Psychoanalyse eine tolle Sache finden, wieso könnten die nicht in ein Seminar kommen, wo man nur Psychoanalyse macht. Und sich da für Psychoanalyse autorisieren, wieso müssen wir das auch noch anbieten?

SvA: Wegen der Mehrheit.

PP: Ja, aber welche Mehrheit? 
SvA: An der TV.

PP: Aber das ist eine Mehrheit, die unter falschen Bedingungen am Seminar ist. Die immer schon meinte, das sei eine Therapie-Ausbildung und eigentlich das will. Und nicht die Psychoanalyse will. Sonst hätten wir ein viel kleineres Seminar, wo Leute sich treffen, die wirklich Psychoanalyse wollen. Und da könnten Therapeuten von allen Richtungen kommen. Die Studierenden könnten sich dann hier wirklich mit der Psychoanalyse auseinandersetzen, aber mit nichts anderem. Das wäre von mir aus gesehen schon rein ästhetisch viel befriedigender.

Journal: Nocheineletzte Frage.Freud schwebte eine psychoanalytische Hochschule vor. Und die Frage wäre jetzt, jenseits dieser Anwendung der Psychoanalyse auf die Psychotherapie und der daraus resultierenden Weiterbildungen in psychoanalytisch orientierter Psychotherapie, was macht das PSZ, um in seinem Kreis eine psychoanalytisch orientierte Hochschule zu entwickeln. Der Preis, den ihr gestiftet hat, geht ja in diese Richtung, weil er sich interdisziplinär versteht.

PP: Wenn man eine Hochschule machen will, dann muss man die Psychoanalyse von der Klinik abkoppeln. Zum Beispiel hat Laplanche an der Universität in Paris eine psychoanalytische Fakultät gegründet. Aber da ist natürlich klar, dass man das völlig abtrennt, dann ist das eine akademische Disziplin, wo jeder, der die Voraussetzungen für die Universität erfüllt, auch teilnehmen kann. Man darf da keine eigene Analyse fordern, darf das nicht auf die Klinik ausrichten. Dann geht es um die Theorie und darum, was die Theorie zur Praxis der Psychoanalyse zu sagen hat. Das ist dann universal, da können alle mitmachen.

SvA: Man kann doch nicht sagen, dass wir das am PSZ nicht mehr haben! Ist es so, dass wegen dieser Psychotherapie-Weiterbildung nicht mehr alle kommen können, nicht mehr mitdiskutieren können, sprechen können? Ist es so? Es ist doch nicht so, wir haben das nach wie vor!

PP: Ja, aber de facto gibt es viele Leute, die nur um der Psychoanalyse willen kommen.

ML: Ja.

SvA: Ja, aber das gibt es sicher! 
PP: Nicht für eine Ausbildung, nein.

SvA: Aber es gibt doch viele Leute, die in die Freitagsseminarien kommen, die in theoretische Vorlesungen kommen, die ...

ML: Es ist etwa die Hälfte, aus den letzten Zahlen, die ich zusammengerechnet habe, etwa die Hälfte, die aus Interesse kommt. Die Kurse besucht.

Journal: Habe ich das richtig verstanden? Nach den Umfragen, die du gemacht hast, würde etwa die Hälfte aus Interesse an der Psychoanalyse ins PSZ kommen und nicht für die Weiterbildung in Psychotherapie.

ML: Ja.

SvA: Und zum Teil nicht mal für Berufliches.

ML: Ja. Das ist so. Es sind mehr TeilnehmerInnen, die Kurse besuchen und aus Interesse kommen, als solche, die für die Weiterbildung kommen.

SvA: Und ich glaube, auch Leute, die nicht therapeutisch arbeiten, die nicht psychoanalytisch in der Praxis arbeiten.

PP: Eigentliche Laien-Analyse.

SvA: Ja, eigentliche Laien, die von anderen Gebieten kommen.

PP: Es gibt ja zwei Stufen des Laien-Analytikers: Der Laie, der Therapie macht und kein Arzt ist, und derjenige, der sich gar nicht hinter eine Couch setzen will, aber der mit der Psychoanalyse sehr viel anfangen kann.

ML: Es sind zum Beispiel Soziologen, Journalisten, SozialarbeiterInnen etc., die aus Interesse kommen, einfach so.

PP: Das kommt dem Begriff der Hochschule nahe ...

SvA: Darum meine ich, dass wir das ein Stück weit realisiert haben ... 
ML: Ich würde sagen, dass wir das zu einem guten Teil am PSZ noch haben, und dass man für diese Leute weiterhin offen bleiben und das weiter pflegen muss.

PP: Das ist ja wunderbar.

Journal: Vielen Dank für dieses Gespräch. 